\title{
Quantification of the second-order nonlinear susceptibility of collagen I using a laser scanning microscope
}

\author{
Arne Erikson \\ Norwegian University of Science and Technology \\ Department of Physics \\ Høgskoleringen 5 \\ 7491 Trondheim, Norway
}

\section{Jonas Örtegren}

Mid-Sweden University

Digital Printing Center

Järnvägsgatan 3

89118 Örnsköldsvik, Sweden

\section{Tord Hompland \\ Catharina de Lange Davies \\ Mikael Lindgren}

Norwegian University of Science and Technology

Department of Physics

Høgskoleringen 5

7491 Trondheim, Norway

\begin{abstract}
Characteristic changes in the organization of fibrillar collagen can potentially serve as an early diagnostic marker in various pathological processes. Tissue types containing collagen I can be probed by pulsed high-intensity laser radiation, thereby generating second harmonic light that provides information about the composition and structure at a microscopic level. A technique was developed to determine the essential second harmonic generation (SHG) parameters in a laser scanning microscope setup. A rat-tail tendon frozen section was rotated in the $x y$-plane with the pulsed laser light propagating along the $z$-axis. By analyzing the generated second harmonic light in the forward direction with parallel and crossed polarizer relative to the polarization of the excitation laser beam, the second-order nonlinear optical susceptibilities of the collagen fiber were determined. Systematic variations in SHG response between ordered and less ordered structures were recorded and evaluated. A $500 \mu \mathrm{m}$-thick z-cut lithiumniobate $\left(\mathrm{LiNbO}_{3}\right)$ was used as reference. The method was applied on frozen sections of malignant melanoma and normal skin tissue. Significant differences were found in the values of $d_{22}$, indicating that this parameter has a potential role in differentiating between normal and pathological processes. @ 2007 Society of Photo-Optical Instrumentation Engineers. [DOI: 10.1117/1.2772311]
\end{abstract}

Keywords: second harmonic generation; scanning microscopy; polarization; tissues.

Paper 07001R received Jan. 3, 2007; revised manuscript received Mar. 21, 2007; accepted for publication Mar. 27, 2007; published online Sep. 5, 2007.

\section{Introduction}

Collagen is the most abundant structural protein in higher vertebrates, and the structure of extracellular collagen plays an important role in various pathological processes and diseases, such as cancer, aging, and wound healing, ${ }^{1,2}$ as well as in drug delivery. ${ }^{3,4}$ Characteristic changes in the organization of fibrillar collagen are known to occur in several diseases and could potentially serve as an early diagnostic marker. Collagen interacts with other connective tissue elements, and changes in the structure of collagen have an impact on the overall structure of the extracellular matrix. There is therefore a great need for improved methods to study the structure of the collagen network. Collagen has a highly crystalline triplehelix structure that is not centrosymmetric, and the molecules are organized on the scale of the wavelength of light. Thus, collagen satisfies the criteria for generating the second harmonic signal, which may be used to image and analyze the collagen network. This has recently been done in a number of cases both in vivo and ex vivo. ${ }^{5-9}$

Address all correspondence to Arne Erikson, Physics Norwegian University of Science and Technology, Høgskoleringen 5-Trondheim, Sør Trøndelag 7491 Norway; Phone: +4773593634; Fax: +4773597710; E-mail address: arne.erikson@phys.ntu.no
Second harmonic generation (SHG) is an optically nonlinear coherent process where two incident photons of frequency $\omega$ are converted into a single photon of twice the frequency $2 \omega .{ }^{10}$ The second-order susceptibility $\chi_{i j k}^{(2)}$ is a third-rank tensor whose elements sum to zero for a material with inversion symmetry. It determines the induced second-order polarization $P_{i}^{(2 \omega)}$ of the material by the electrical field projected along $E_{j}^{(\omega)} E_{k}^{(\omega)}$ and may represent a quantitative measurement of SHG. Collagen and other non-centrosymmetric molecules such as microtubuli and myosin as well as interfaces between two media are able to generate as SHG signal. ${ }^{11-14}$ Based on the SHG signal, it is possible to image such molecules without any exogenous labeling. Using multiphoton scanning microscopy, these molecules may be colocalized with other cellular parameters based on their endogenous fluorescence or specifically labeled with fluorophores. Multiphoton microscopy has the advantage of improved signal-to-background ratio and imaging at greater depths than confocal laser scanning microscopy. ${ }^{7}$ Despite similarities, SHG and two-photon excited fluorescence are based on fundamentally different phenomena. SHG is associated with a coherent nonlinear scattering process, whereas two-photon excited fluorescence relies

1083-3668/2007/12(4)/044002/10/\$25.00 @ 2007 SPIE 
on nonlinear absorption followed by fluorescence emission, i.e., the emitted photons are normally not coherent with the absorbed ones. However, when combined, the two measurement modes provide an important tool for imaging tissue intravitally in vivo or in sections. ${ }^{1,7,15,16}$

In the present work, a method to quantify the collagen structure by its second-order susceptibility in tissue was developed and exploited. The procedure is carried out in a laser scanning microscope setup, thus allowing us to extract SHGrelevant parameters in the same sample configuration as used for imaging (or other relevant spectroscopic fluorescence microscopy characterization). Based on measuring the elements of the matrix describing the nonlinear susceptibility, a quantitative parameter representing the SHG signal was obtained. To our knowledge, this quantitative parameter has not been previously exploited in biomedical applications. Such measurements may provide unique fingerprint data and be of diagnostic value in assessing normal versus pathological conditions, as was demonstrated for tissue samples from normal skin and malignant melanoma.

\section{Materials and Methods}

\subsection{Experimental Setup}

The measurements were performed using a laser scanning microscope (Axiovert 100M, LSM 510, Zeiss, Germany) with a C-Apochromat $10 \times / 0.45$ water immersion objective for rattail tendon (RTT) samples or a Plan-Neofluar $20 \times / 0.5$ I for melanoma and normal skin samples. The laser source was a mode-locked Ti:Sapphire laser (Mira Model 900-F, Coherent, Inc., Laser Group, Santa Clara, CA) pumped with a $5 \mathrm{~W}$ Verdi laser. The SHG signal intensity was investigated in the 740-900 nm spectral interval, and for RTT the SHG intensity increased at shorter wavelengths, as also shown by others. ${ }^{17}$ For tumor tissue, the optimal excitation wavelength was found in the range of 800 to $810 \mathrm{~nm}$. Thus, the RTT and the skinmelanoma samples were excited at $\lambda=780 \mathrm{~nm}$ and $\lambda$ $=810 \mathrm{~nm}$, respectively, with a pulsewidth of approximately $180-200 \mathrm{fs}$ at the $76 \mathrm{MHz}$ repetition rate. Higher excitation powers were used for the skinmelanoma samples than for RTT. The laser was configured to give linearly polarized light in the east-west direction. A rotation table was constructed in which the sample was rotated in the $x y$-plane with the pump laser light propagating along the $z$-axis. The sample was rotated one full rotation, and the SHG intensity was recorded at specific angular increments (typically each $10 \mathrm{deg}$ ). A linear polarizer (analyzer) was placed after the sample, between the condenser and the detector, either perpendicular (north-south) or parallel (east-west) to the linearly polarized laser light when using RTT, but only parallel when characterizing the other samples. A bandpass filter (385-425 nm) was placed in front of the detector to remove the residual light of the pump beam. The forward-generated SHG light was detected using a photomultiplier tube. Detector gain and laser power varied and were set to optimize the SHG signal from the crystalskinmelanoma.

A region of interest (ROI) was selected on the recorded images for each rotation angle of the sample. Various sizes and shapes of ROIs were tested; after thresholding to remove black pixels, no significant differences were found in the re- sults. The intensity data of the ROI were loaded into Matlab (The Math Works, Natick, MA) and further processed using customized analysis software.

We also attempted to vary the azimuthal angle between the sample and the electric field of the linearly polarized pump laser light by keeping the sample at a constant angle and instead using a half-wave plate at certain angular orientations to vary the polarization direction of the pump beam. However, this strategy was abandoned since it resulted in certain artifacts due to ellipticity introduced in the galvanometric mirrors and dichroic beamsplitter, as also noted by others. ${ }^{18}$

\subsection{Sample Preparation}

A $500 \mu \mathrm{m} z$-cut single-crystal $\mathrm{LiNbO}_{3}$ sample (1691-5 Inrad, Northvale, NJ) of known second-order nonlinear susceptibility was used as a reference standard. Care was made to determine the essential beam parameters such as Rayleigh range and polarization, to be described ahead. The absolute value of the second-order nonlinear susceptibility describing SHG from the collagen sample could then be determined by comparison with the SHG signal of $\mathrm{LiNbO}_{3}$ placed at the very same position using the same focusing geometry of the excitation laser beam.

RTT from 5-6 month-old Sprague-Dawley (female) rats was used as a primary collagen sample. Human melanoma Xenografts were grown subcutaneously in the leg in 4-6week-old female athymic BALB/c-nu/nu mice (Taconic M\&B, Denmark) by injecting a $30 \mu \mathrm{l}$ suspension of $2 \times 10^{6}$ human melanoma cells from the cell line FME. ${ }^{19}$ The mice were anesthetized by subcutaneous injection of Fentanyl/Midazolam/Haldol/sterile water (3:3:2:4) at $10 \mathrm{ml} / \mathrm{kg}$ bodyweight (Hameln Pharmaceuticals, Germany; Alpharma AS, Norway; and Janssen-Cilag AS, Norway). The xenografts were grown for 3-6 weeks, and the tumor size ranged from 500 to $1000 \mathrm{~mm}^{3}$. The animals were kept under pathogen-free conditions at a constant temperature of $24-26^{\circ} \mathrm{C}$ and at humidity of $30-50 \%$ and allowed food and water ad libitum. All animal experiments were carried out with ethical committee approval. The mice were sacrificed by cervical dislocation and the tumors were excised. Samples were obtained approximately $800 \mu \mathrm{m}$ from the tumor periphery. Mouse skin biopsies (dermis) served as normal tissue samples.

All samples were embedded in Tissue Tec (O.C.T., Histolab Products, Göteborg, Sweden) and frozen in liquid nitrogen. Frozen sections, $5 \mu \mathrm{m}$ thick, were mounted on glass slides and stored at $-80^{\circ} \mathrm{C}$.

\subsection{Analysis of SHG: General Considerations}

The theory of SHG and nonlinear optics ${ }^{10}$ is well known, and extensive literature exists on the subject. The concepts relevant for our analysis will be briefly reviewed here. The induced polarization of a medium subjected to an intense electromagnetic field such as an intense laser pulse can be expressed in a power series of the field strength $E_{i}(i, j, k$ are Cartesian components): 


$$
P_{i}=\varepsilon_{0} \chi_{i j}^{(1)} E_{j}+\varepsilon_{0} \chi_{i j k}^{(2)} E_{j} E_{k}+\varepsilon_{0} \chi_{i j k l}^{(3)} E_{j} E_{k} E_{l}+,
$$

where $P_{i}$ is the $i$ th component of the induced polarization, $\varepsilon_{0}$ is the vacuum permittivity, and $\chi_{i j}^{(n)}$ denotes the $n$ th-order susceptibility and is a tensor of rank corresponding to the number of subscripts, i.e., $\chi_{i j k}^{(2)}$ is termed the second-order nonlinear susceptibility and is a third-rank tensor. $\chi_{i j k}^{(2)}$ can be expressed by the third-rank $d$-tensor given by $d_{i j k}=\chi_{i j k}^{(2)} / 2$, and the effective $d$-value is written as $d_{\mathrm{eff}}=\hat{e} \tilde{d}$ : $\hat{e} \hat{e}$, where $\hat{e}$ is a unit vector describing the electric field or polarization field of the light wave $(\bar{E}=\hat{e} E)$. The tensor related to $\mathrm{SHG}, \chi_{i j k}^{(2)}$, reflects the symmetry and nonlinear optical properties of the material. Due to symmetry selection rules, it is found that the elements of the tensor $\chi_{i j k}^{(2)}$ sum to zero for a material with inversion symmetry. It is common practice to use contracted notation in order to describe the second-order susceptibility. ${ }^{10}$ The notation $i, j, k$ is then altered to $i, l$, where $l$ represents the propagation of the fundamental beam (excitation light) along the principal axes of the nonlinear media. This notation will be used ahead.

Assuming a focused Gaussian laser beam, the expression for the SHG light intensity, $I_{2 \omega}$, is given as ${ }^{10,20,21}$

$$
I_{2 \omega}=\frac{p}{n_{2 \omega} n_{\omega}^{2}}\left(I_{\omega}\right)^{2} d_{\mathrm{eff}}^{2}\left(\int_{z_{0}}^{z_{0}+L} \frac{e^{i \Delta k z}}{1+i z / z_{R}} d z\right)^{2},
$$

where $p$ is a parameter containing fundamental constants and certain beam quality parameters, $z_{R}$ is the Rayleigh range, $I_{\omega}$ is the laser light intensity, $n_{m \omega}$ is the refractive index at frequency $m \omega(m=1,2), d_{\text {eff }}$ is the effective second-order nonlinear susceptibility, and $\Delta k=4 \pi(\lambda)^{-1}\left(n_{\omega}-n_{2 \omega}\right)$ is the phase mismatch. The second harmonic intensity was measured for an $\mathrm{LiNbO}_{3}$ crystal with known $d_{\text {eff }}$ and thickness $L$ and compared with the second harmonic intensity in collagen in RTT or in other samples. The second-order nonlinear susceptibility for collagen could then be determined.

\subsection{Analysis of the Reference Sample $\mathrm{LiNbO}_{3}$}

The $d$ matrix describing the SHG in $z$-cut $\mathrm{LiNbO}_{3}$, having $3 m$ symmetry along the $z$-axis, is given in, e.g., Ref. 10. For the case of the laser beam propagating along the $z$-axis and the analyzer oriented perpendicular to the polarization direction of the laser light (crossed polarizers), $d_{\mathrm{eff}}$ is

$$
d_{\mathrm{eff}}=-d_{22} \cos 3 \beta,
$$

where $\beta$ is the azimuthal angle between one crystallographic axis and the electric field of the laser beam. Consequently, the highest value of $d_{\text {eff }}$ for $z$-cut $\mathrm{LiNbO}_{3}$ is simply $d_{\text {eff }}=\left|d_{22}\right|$ in this configuration (e.g., for $\beta=0$ degree $+m 60$ degree). If we rotate the crystal about the $z$-axis, the effective $d$-tensor has three-fold symmetry. Each lobe generates a positive and negative maximum such that the measured intensity [square of Eq. (3)] is in practice generating a six-fold symmetry for a full revolution (data not shown). The light power of the SHG signal was calculated by approximating the envelope function in Eq. (2) by the sum

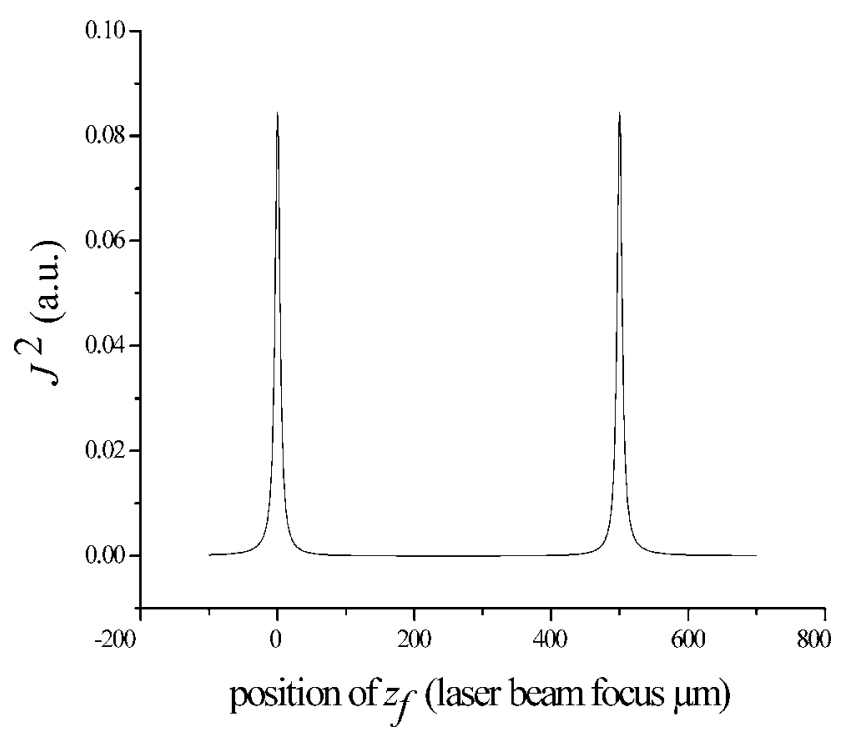

Fig. 1 Theoretical plot of last term in Eq. (2) $\left(=\rho^{2}\right)$ as focus of laser beam is moved along the $z$-axis through a $500 \mu \mathrm{m} \mathrm{LiNbO}_{3}$ sample using a Rayleigh range $z_{R}=14 \mu \mathrm{m}$ (see below). Parameter values: $\lambda$ $=780 \mathrm{~nm} ; \Delta n=-0.2 ; n=2.26$.

$$
\int_{-z}^{z} \frac{e^{i \Delta k z}}{1+i z / z_{R}} d z \approx \sum_{\substack{z=-1000 \\ \Delta z=0.01}}^{z=1000} \frac{e^{i \Delta k z}}{1+i z / z_{R}} \Delta z
$$

A Matlab integration routine was used separately to verify that the number of steps used in the approximation was appropriate. Thus, Eq. (4) was used with Eq. (2) to calculate the generated SHG intensity for a given experimental situation.

As an example, Fig. 1 shows the simulated SHG light power when $\mathrm{LiNbO}_{3}$ is scanned in the positive $z$-direction through the focus $(z=0)$ of the laser beam. As the surface of the sample reaches the focal plane, Eq. (4) reaches a maximum value. As the sample continues to be moved in the positive $z$-direction, Eq. (4) becomes small again, since the focus of the laser beam now is inside the sample, and $\mathrm{SH}$ light generated before and after focus are phase shifted by $\pi$ radians (Gouy phase shift ${ }^{10}$ ). The function again reaches a second maximum as the upper surface of the sample reaches focus.

\subsection{Determination of Pump Laser Beam Parameters}

Using Eqs. (2) and (4), it was possible to determine laser beam parameters necessary to estimate the $d$-coefficient of collagen. The full width at half-maximum (FWHM) of the peak increases with increasing Rayleigh range, $z_{R}$, thereby allowing the determination of $z_{R}$ of the laser beam by recording SHG light as the sample is moved in the $z$-direction. ${ }^{20}$ The Rayleigh range, $z_{R}$, was determined for the objectives used by stepping through a $500 \mu$ m-thick $z$-cut $\mathrm{LiNbO}_{3}$ sample through focus and simultaneously detecting SHG intensity $(z$ scan step). The last term of Eq. (2) was subsequently fitted to experimental data. A Rayleigh range of $14 \mu \mathrm{m}$ gave a reasonable fit for the $10 \times$ objective, as shown in Fig. 2, and a value of $10 \mu \mathrm{m}$ was found for the $20 \times$ objective. 


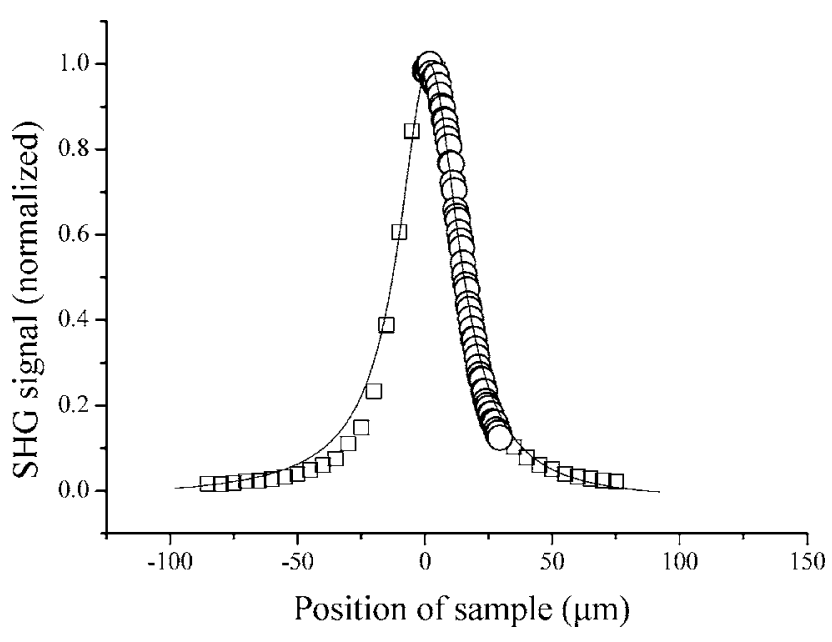

Fig. $2 \mathrm{SHG}$ signal as the $\mathrm{LiNbO}_{3}$ sample is scanned in the $z$-direction through focus in order to determine the Rayleigh range $z_{R}$ of the laser beam. Squares represent data points spaced $5 \mu \mathrm{m}$ apart. Circles represent data points spaced $0.5 \mu \mathrm{m}$ apart. The full curve is the last term of Eq. (2) with $z_{R}=14 \mu \mathrm{m}$. ( $z_{R}$ is determined by the full width at half-maximum). A $10 \times$ objective was used.

\subsection{Analysis of SHG of Collagen}

The $d$-matrix describing SHG in collagen is written as

$$
d_{\mathrm{eff}}=\left[\begin{array}{ll}
\hat{e}_{1} \hat{e}_{2} \hat{e}_{3}
\end{array}\right]\left[\begin{array}{cccccc}
0 & 0 & 0 & 0 & 0 & d_{16} \\
d_{21} & d_{22} & d_{23} & 0 & 0 & 0 \\
0 & 0 & 0 & d_{34} & 0 & 0
\end{array}\right]\left[\begin{array}{c}
\hat{e}_{1}^{2} \\
\hat{e}_{2}^{2} \\
\hat{e}_{3}^{2} \\
2 \hat{e}_{2} \hat{e}_{3} \\
2 \hat{e}_{3} \hat{e}_{1} \\
2 \hat{e}_{1} \hat{e}_{2}
\end{array}\right] .
$$

The unit vectors $\hat{e}_{1}, \hat{e}_{2}$, and $\hat{e}_{3}$ relate the coordinate system of the laser beam electric field or the polarization field of the light wave $(\bar{E}=\hat{e} E)$ to the collagen fiber. The $6 \times 1$ and the $1 \times 3$ matrices in Eq. (5) describe the generating field and the generated field, respectively. Referring to Fig. 3 (model 1),

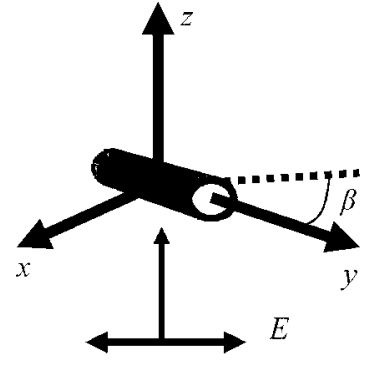

Model 1

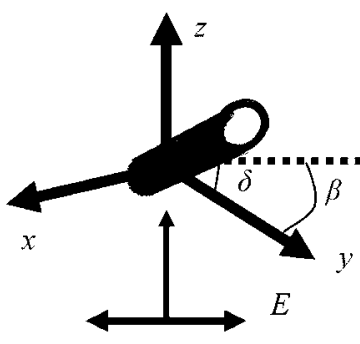

Model 2
Fig. 3 Definition of the coordinate system for the collagen fiber: The angle $\beta$ is the angle in the $x y$-plane between the fiber axis and the electric field. The angle $\delta$ is the angle between the fiber and the $x y$-plane. $C y l i n d e r=$ collagen fiber; $E=$ electric field of laser light polarized along the $y$-axis and propagating in the $z$-direction. the polarization of the pump electric field of the laser is described by $\left(\hat{e}_{1}, \hat{e}_{2}, \hat{e}_{3}\right)=(-\sin \beta, \cos \beta, 0)$. Using parallel polarization (east-west) in relation to the laser beam, the generated SHG polarization is described with the same unit vector. In accordance with previous approaches, ${ }^{20,22}$ collagen has $C_{\infty} m m$-symmetry along the fiber (the $y$-axis, Fig. 3). Cylindrical symmetry $(x=z)$ implies that $d_{16}=d_{34}$ and $d_{21}=d_{23}$, and Kleinman symmetry in addition gives $d_{16}=d_{21}$. The effects of symmetry conditions are further discussed in the sections ahead. Two models were used to characterize the fiber orientation. In the simpler model 1 , it was assumed that the fiber was positioned with the long axis entirely in the $x y$-plane. We also examined the case when the fiber was tilted an angle $\delta$ out from the $x y$-plane, model 2. Cartoons of the models along with the coordinate systems associated with the models are depicted in Fig. 3. The general expression for SHG light produced with parallel polarization in relation to the pump beam is then given by

$d_{\text {eff }}^{2^{\prime}}=\left[3 d_{16}\left(\cos \beta \cos \delta-\cos ^{3} \beta \cos ^{3} \delta\right)+d_{22} \cos ^{3} \beta \cos ^{3} \delta\right]^{2}$.

For the SHG measured with crossed polarizers with respect to the pump beam, one obtains in an analogous manner

$$
\begin{aligned}
d_{\mathrm{eff}_{\perp}}^{2^{\prime}}= & {\left[d_{16}\left(3 \sin \beta \cos ^{2} \beta \cos ^{3} \delta-\sin \beta \cos \delta\right)\right.} \\
& \left.-d_{22} \sin \beta \cos ^{2} \beta \cos ^{3} \delta\right]^{2} .
\end{aligned}
$$

In these expressions Kleinman symmetry was assumed. To get the expression for model 1, $\delta$ in Eqs. (6) and (7) is set to zero. [The detailed derivations of Eqs. (6) and (7) are shown in the Appendix.]

Some brief remarks should be made concerning the focused beam. A paraxial approximation is assumed in the model used, meaning that the polarization of the laser light is unchanged in the focus. Using an objective with a numerical aperture of 0.45 , this approximation does seem reasonable., ${ }^{9,20}$ Moreover, with a tight focus, the driving field will have a wide range of propagation directions in addition to a greatly enhanced intensity near the focal center. These additional polarization components are not considered in the model used, an approximation that may be justified due to the moderately low numerical aperture of the objective used in the experiments. There is a phase lag of $\pi$ as the focused light beam travels through its focal center known as the Gouy shift or phase anomaly. ${ }^{23}$ The consequence of this is that the SHG signal from a focused beam propagates off-axis in two welldefined symmetric lobes. Some SHG radiation may also occur in the backward direction depending on the molecular distribution in the SHG active volume. ${ }^{13}$ The collection optics should thus have a numerical aperture no smaller than that of the excitation optics. This condition was fulfilled in the used experimental setup [numerical aperture 0.55 and 0.45 $(10 \times), 0.5(20 \times)$ for the collection and excitation optics, respectively].

\subsection{Statistical Analysis}

The statistical significance between data was determined by a two-sample $t$ test, and all the statistical analyses were per- 


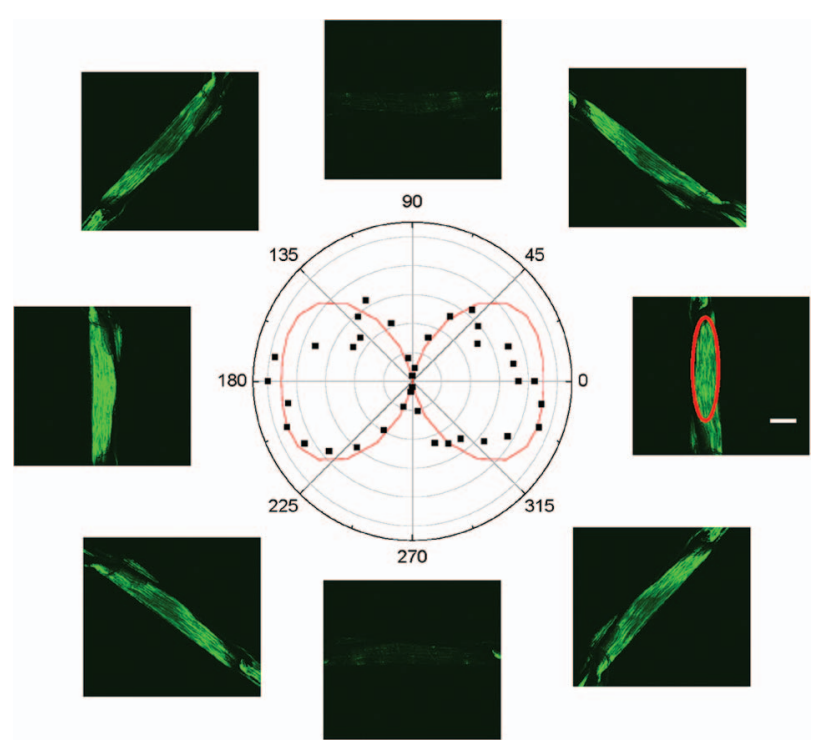

Fig. 4 Experimental SHG data as a function of azimuthal angle $\beta$ (black points) and best-fitted curve [red line; Eq. (6)] where the analyzer is parallel (II) to the polarization of the excitation laser light. The concentric circles (light gray) indicate a linear scale of SHG signal intensity. A few representative images showing RTT collagen are placed at corresponding angles where data were collected. Bar $=100 \mu \mathrm{m}$. The red outline in one of the SHG images indicates a chosen ROI.

formed using the significance criterion of $P=0.05$ (Minitab, Minitab Inc., State College, PA). As a quantitative measure of agreement between experimental data and fit, the squared standard error of the estimate was used:

$$
\sum_{i}\left(\text { data }_{i}-\mathrm{fit}_{i}\right)^{2} / n
$$

\section{Results and Discussion}

\subsection{General Appearance of SHG Images}

Typical SHG images of well-ordered collagen sections of RTT are shown in Figs. 4 and 5. The sample was rotated between parallel (Fig. 4) and crossed polarizers (Fig. 5). An ROI was selected, and the total intensity of that ROI was calculated for each SHG image. The polar plot in the center of Figs. 4 and 5 shows how the total integrated SHG intensity varies with sample orientation $(\beta)$ for each given polarization combination. Equations (6) and (7) with $\delta=0$ were fitted to experimental data as shown in the polar plots of Figs. 4 and 5. Equation (8) gave the value 0.03 for both parallel and crossed polarizers. An overall trend is seen; two maxima occur in the plot for the case of parallel polarizers, whereas for the case of crossed polarizers, four maxima occur. In a number of tumors, abnormal collagen fibril aggregates occur, manifesting themselves as wide variations in diameter and cross-sectional profile of the collagen fibrils. ${ }^{24}$ A strong SHG signal is produced only by ordered structures, and analysis in terms of magnitude and angular dependence could be used as a method to study the structure of the extracellular matrix. Such analysis may be used to distinguish between normal and malignant tissue and to characterize the influence of treatment in tissues. A prereq-

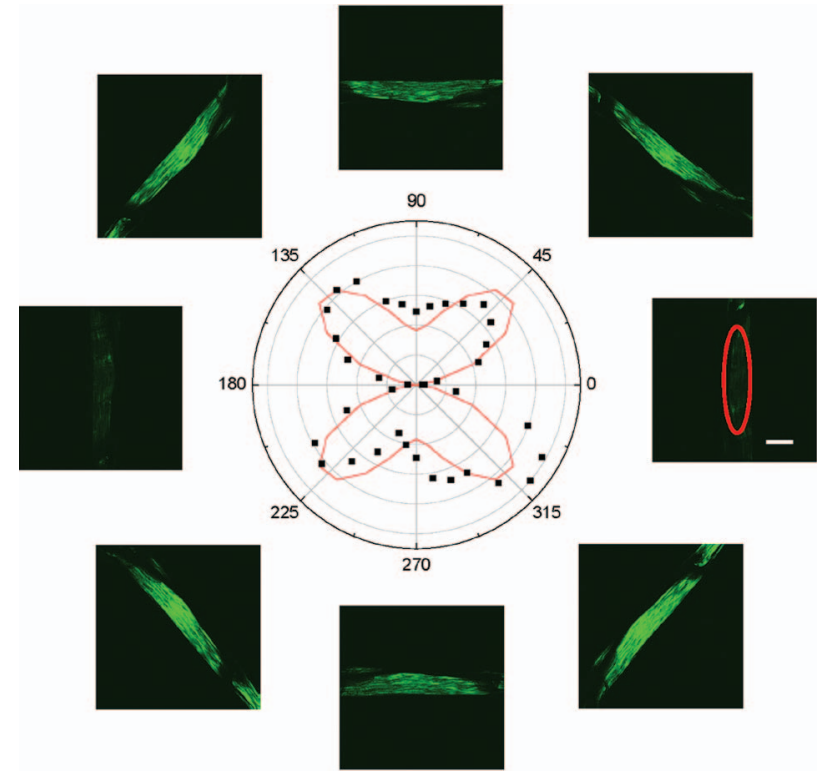

Fig. 5 Experimental SHG data as a function of azimuthal angle $\beta$ (black points) and best-fitted curve [red line; Eq. (7)] where the analyzer is perpendicular $(\perp)$ to the polarization of the excitation laser light. The concentric circles (light gray) indicate SHG signal intensity. A few representative images showing RTT collagen are placed at corresponding angles where data were collected. Bar $=100 \mu \mathrm{m}$. The red outline in one of the SHG images indicates a chosen ROI.

uisite for such studies is, however, that SHG is able to determine variations in the structural order. Therefore, different ROIs in several RTT frozen sections were analyzed and classified as either more ordered or less ordered, based upon a subjective visual evaluation of each ROI. Representative im-
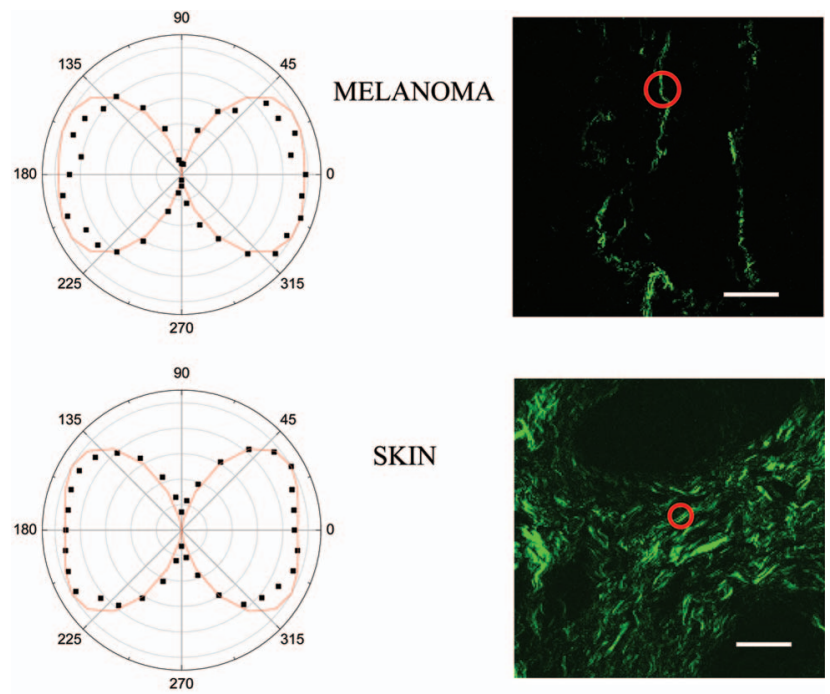

Fig. 9 Typical polar plots of regions with ordered collagen fibrils in malignant human melanoma and in normal mouse skin. Data were obtained with the analyzer parallel to the polarization of the excitation laser light. The concentric circles (light gray) indicate SHG signal intensity. Images shown are of SHG signals from corresponding sections of melanoma and skin. Bar=50 $\mu \mathrm{m}$. The red circle in the SHG images indicates a chosen ROI. 


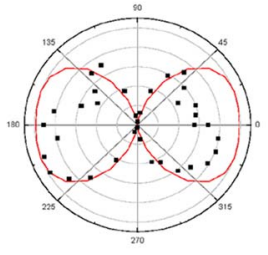

ROI 1 ||

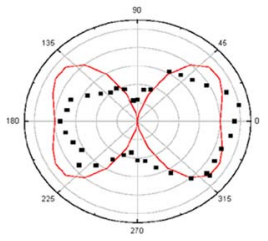

ROI 2 ||

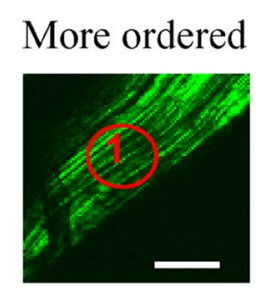

Less ordered
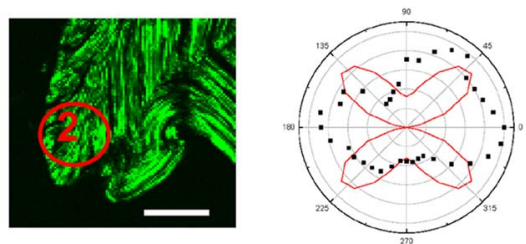

ROI $2 \perp$
Fig. 6 Typical polar plots of regions with more ordered and less ordered collagen fibrils in RTT collagen. $\|$ and $\perp$ symbolize that the analyzer is parallel and perpendicular to the polarization of the excitation laser light, respectively. The concentric circles (light gray) indicate SHG signal intensity. Bar $=50 \mu \mathrm{m}$.

ages and data points are shown in Fig. 6. The ROIs with the more ordered (data equivalent to those in Figs. 4 and 5) and less ordered fibril structure are denoted ROI 1 and ROI 2 in Fig. 6, respectively. Equation (8) gives the values 0.03 and 0.06 for ROI 1 with parallel and crossed polarizers, respectively. Compared with ROI 2, these values were 0.06 and 0.18 , thus showing larger discrepancies between fit and data for this case. Equations (6) and (7) describe $\mathrm{SH}$ generated from a monocrystalline structure, and the structure in ROI 2 of Fig. 6 is obviously far from monocrystalline, thereby explaining the discrepancies. Data points generated from the less ordered fibril structures could possibly be useful to deter- mine the distribution of the orientation of the fiber segments and to yield a measure of the structural order. It would, however, be necessary to know the relation between $d_{22}$ and $d_{16}$ beforehand. From a qualitative evaluation it can be concluded that the angular dependence of the SHG signal varies in a manner related to the structural order of the fibrils. This finding could be valuable to characterize the fibril structure and possible changes in this structure.

\subsection{Simulations of Typical d-Tensor Contributions}

Simulations of Eqs. (6) and (7) were made in order to examine the contributions from the various d-tensor elements for a well-defined crystalline order. Although the simulations in Figs. 4 and 5 are in more or less agreement with experimental data, various refinements of the most naïve models were made and tested. Figs. 7(a) and 7(b) represent the isolated contribution from $d_{16}$ and $d_{22}$, respectively, for the case of parallel polarizers as the angle $\delta$ in Eq. (6) is varied, corresponding to a fiber axis that is tilted with respect to the $x y$-plane. The polar plot of $d_{16}$ shows a distinct difference in shape upon varying $\delta$. However, upon combining the contributions from $d_{22}$ and $d_{16}$ and varying the quotient between the two in a polar plot, similar variations in the shape of the polar curve are observed. It is consequently difficult to estimate the angle $\delta$ based on the experimental data. It is reasonable, however, to assume that $\delta$ is small (less than $10 \mathrm{deg}$.) for most samples, and since the influence of $\delta$ on the simulations is minor for those cases, the quotient between $d_{22}$ and $d_{16}$ may still be estimated with some certainty. The angle $\delta$ does not play a significant role when the analysis is based upon frozen sections of RTT. The introduction of $\delta$ may, however, be more relevant for analysis of SHG data from frozen sections of tumor tissues and various biopsies, where $\delta$ depends on the orientation of the tissue during preparation of the frozen sections. Due to the randomness where the sectional planes lie in a spherically shaped biopsy, it is very likely in such cases that

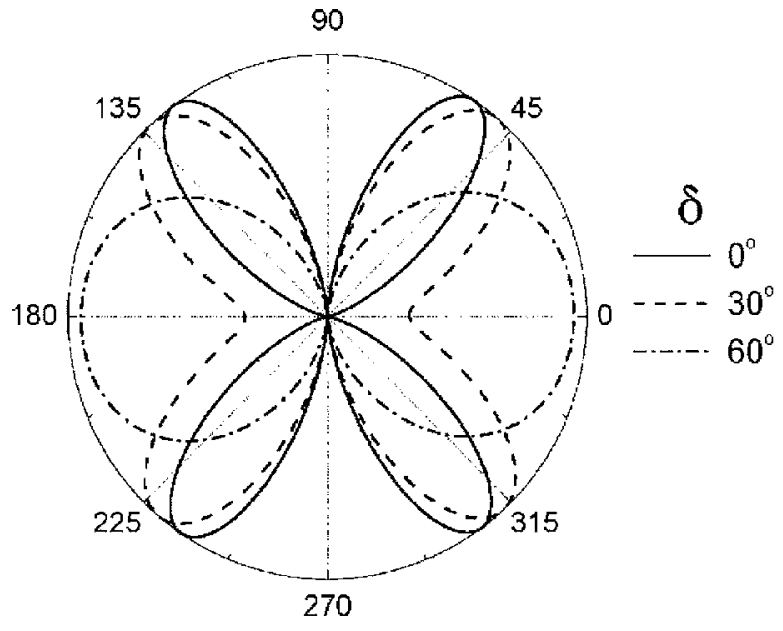

(a)

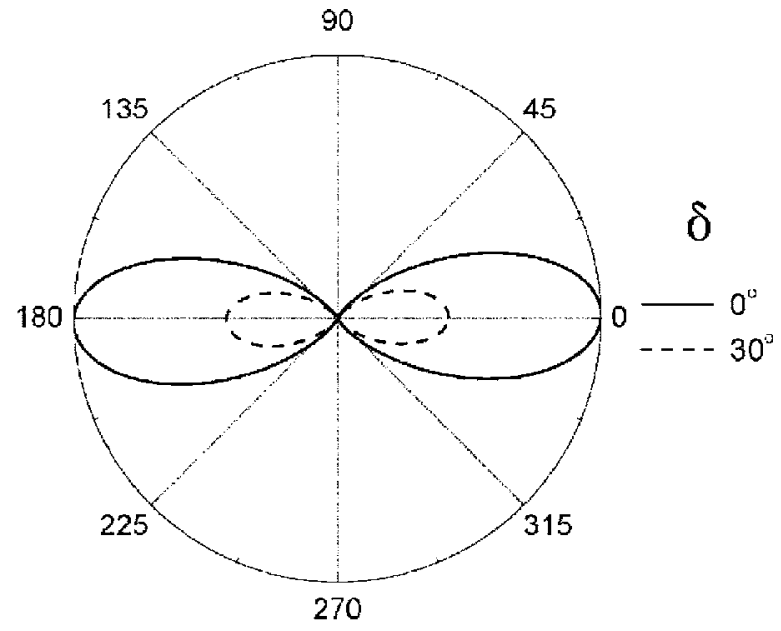

(b)

Fig. 7 Contribution of $d_{16}$ (a) and $d_{22}$ (b) to the SHG light intensity as the fiber is tilted above the $x y$-plane, using parallel polarizers. The angle $\delta$ is the angle between the $x y$-plane and the fiber axis. 
the collagen fiber axis may be severely tilted with respect to the $x y$-plane.

\subsection{Collagen Structure and Symmetry Approximation}

Type I collagen is a triple helix about $300 \mathrm{~nm}$ in length and $1.5 \mathrm{~nm}$ wide, and some authors have proposed it as having a quasi-hexagonal symmetry. ${ }^{25}$ The assembly of collagen I into fibrillar aggregates involves multiple steps, essentially being a self-driven process. A hierarchical order is established, and the structures are organized from molecules to microfibrils, fibrils, and fascicles. Microfibrils consist of five or six individual collagen molecules arranged into a bundle with a diameter of about $4 \mathrm{~nm}$. The microfibrils may then twist around each other to form a larger bundle called a fibril. The fibril can be characterized as a suprahelical structure with a diameter ranging from 20 to $500 \mathrm{~nm} .^{26,27}$ Fibrils may be further organized into structures called fascicles and organized into parallel bundles. From the complexity of the arrangement, it follows that the underlying symmetry is, most likely, lost at the length scale studied here, and the collagen fibers can therefore be approximated as having cylindrical symmetry. This assumption can be further justified by the scanned images and the azimuthal angle dependence of the generated SH light presented in this work of the best oriented cases (Figs. 4 and $5)$. The measured nonlinear susceptibility represents an average of local arrangements of the fibrils on a scale too large to reveal spatial arrangements at the microfibrillar level. If there is any underlying hexagonal structure (or similar geometrical configuration), it may well be approximated by a cylindrical model. Furthermore, the assumption of cylindrical symmetry is in accordance with analytic models for SHG in collagen fiber discussed in the literature. ${ }^{20,28,29}$

\subsection{Determination of the $d_{22}$-Coefficient of RTT Collagen}

The $d_{22}$-coefficient describing SHG was determined for RTT collagen by comparison with the SHG signal from an $\mathrm{LiNbO}_{3}$ sample with known $d$-coefficients. The measurements on the $\mathrm{LiNbO}_{3}$ sample were performed on the lower surface of the sample at a fixed azimuthal angle that yielded the largest possible SHG signal. Equations (2) and (3) give

$$
\begin{aligned}
& \left.\frac{d(\beta)^{2}}{I(\beta)_{2 \omega} n_{2 \omega} n_{\omega}^{2}}\left(\int_{0}^{t_{s}} \frac{e^{i \Delta k z}}{1+i z / z_{R}} d z\right)^{2}\right|_{\text {collagen }} \\
& =\left.\frac{d_{22}^{2}}{I_{2 \omega} n_{2 \omega} n_{\omega}^{2}}\left(\int_{0}^{500} \frac{e^{i \Delta k z}}{1+i z / z_{R}} d z\right)^{2}\right|_{\mathrm{LiNbO}_{3}},
\end{aligned}
$$

which was solved for $d(\beta)$. The maximum SHG signal from the RTT sample when using parallel polarizers was found to be at the azimuthal angle $\beta=0 \mathrm{deg}$ or $180 \mathrm{deg}$ (Fig. 4). Thus, when $\delta=0 \mathrm{deg}$, Eq. (6) gives $d_{\text {eff }}^{\prime}=d(\beta=0 \mathrm{deg}, 180 \mathrm{deg})$ $=d_{22} . d_{22}$ was used in all the calculations as an input parameter to determine $d_{16}$. The following data were used; ${ }^{20,30}$ $d_{22, \mathrm{LiNbO}_{3}}=2.76 \mathrm{pmV}^{-1}, \quad n_{\mathrm{LiNbO}_{3}}=2.26 ; \quad \Delta n_{\mathrm{LiNbO}_{3}}=-0.2$; $n_{\text {collagen }}=1.5 ; \quad \Delta n_{\text {collagen }}=-0.03, \quad$ where $\Delta n=n_{\omega}-n_{2 \omega}$, $\lambda_{\omega}=0.78 \mu \mathrm{m}$, and $z_{R}=14 \mu \mathrm{m}$. The $d_{22}$-value of collagen calculated according to Eq. (9), however, becomes strongly dependent on the selected values of the section thickness, $t_{s}$, and

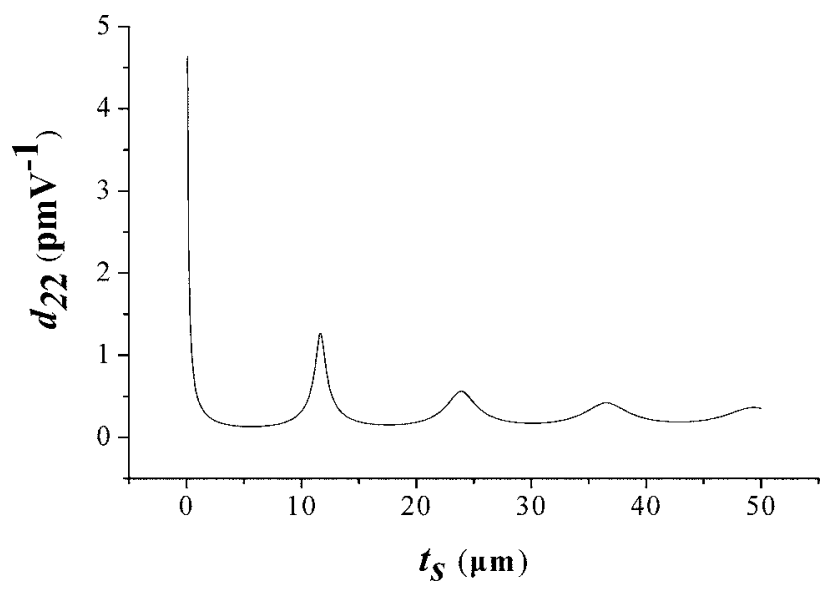

Fig. 8 Calculated $d_{22}$ coefficient of collagen calculated according to Eq. (8) as a function of chosen $t_{s}$.

the phase mismatch, $\Delta k=4 \pi \Delta n \lambda_{\omega}^{-1}$, of the collagen fiber. In the literature, values for $\Delta n=n_{\omega}-n_{2 \omega} \approx-0.03--0.08$ have been reported, ${ }^{31,32}$ where no reference is made to polarization states. The yield of SHG is strongly dependent on the coherence length defined as $l_{c}=\lambda_{\omega}(4 \Delta n)^{-1}$, which equals the thickness of a nonlinear material effective in generating second harmonic radiation. With $\lambda_{\omega}=780 \mathrm{~nm}$ and $\left|\Delta n_{\text {collagen }}\right|=0.03$, one finds $l_{c}=6.5 \mu \mathrm{m}$, which is close to the thickness, $t_{s}$, of the section thickness studied here. Consequently, small variations in the input parameters $t_{s}$ and $\Delta n$ in the simulations have a large influence on the calculated tensor element $d_{22}$. One example is shown in Fig. 8, where the calculated $d_{22}$-coefficient is presented as a function of the input parameter $t_{s}$. Peaks appear periodically in the graph as a consequence of phase mismatch as SH light is generated inside the nonlinear media. A similar dependence was found upon varying the input parameter $\Delta n$ for a fixed $t_{s}$. The section thickness in our experiments was $5 \mu \mathrm{m}$, which corresponded to the sample thickness as the collagen fibers were homogeneously distributed throughout the section thickness. Since $\Delta n$ could not be determined with accuracy, only a lower bound for the $d_{22}$-coefficient of collagen could be determined.

Equation (9) shows that there are other parameters than section thickness and refractive index dispersion that influence the calculation of the $d$-coefficients. The significance of the Rayleigh length $z_{R}$ on the calculated $d$-coefficients was studied separately. This parameter was determined experimentally as $z_{R}=14 \mu \mathrm{m}$ (Fig. 2) for the $10 \times$ objective and $z_{R}=10 \mu \mathrm{m}$ for the $20 \times$ objective used. A range of $8<z_{R}<22 \mu \mathrm{m}$ (thereby including the experimentally determined values) was studied by first calculating the integral squared on the left side of Eq. (9) in this range, fixing $t_{s}$ at either 5 or $10 \mu \mathrm{m}$, and $\Delta n_{\text {collagen }}=-0.03$, and thereafter plotting $d_{22}$ versus the obtained values of that integral squared (data not shown). The largest variations in $d_{22}$ were observed for $t_{s}=10 \mu \mathrm{m}$ (approximately 0.25) as compared with $t_{s}=5 \mu \mathrm{m}$ (approximately 0.025). More accurate values of $d_{22}$ are thus expected for $t_{s}=5 \mu \mathrm{m}$. Three different sections of RTT were rotated $360 \mathrm{deg}$, and SHG intensity signals were collected using parallel and crossed polarizers. Values of $d_{22}$ were calculated according to Eq. (9) for the case of parallel 
Erikson et al.: Quantification of the second-order nonlinear susceptibility of collagen I...

polarizers and an ROI of well-ordered collagen fibers. A lower bound from experiments on the three RTT samples having $t_{s}=5 \mu \mathrm{m}$ was found to be $d_{22}=0.15 \pm 0.01 \mathrm{pmV}^{-1}$, which is in accordance with previous findings. ${ }^{20}$

At a higher level of resolution, there is no general consensus in the literature as to where the SHG signal emanates from. It has been hypothesized that the SHG signal emanates only from the fibril "shell" rather than from its bulk, ${ }^{9}$ where typical fibril diameters are of the order $\lambda_{2 \omega}$, and that this might be a consequence of fibrils being tubelike rather than rodlike. ${ }^{33}$ Another factor influencing SHG light power from the collagen is the relative orientation of neighboring fibrils. Variations in the SHG signal might be due to neighboring collagen fibrils being oriented parallel and antiparallel, either strengthening or weakening the SHG signal, respectively. ${ }^{20,29}$ Consequently, the value of $d_{22}$ reported in this work is the lower bound at the given length scale. At a higher level of resolution, a higher $d_{22}$-value could be expected, as also reported in the literature.

\subsection{Determination of the $d_{16}$ Coefficient in RTT Collagen}

$d_{22}$-values found as described earlier were used as input for generating best-fit values of $d_{16}$ by use of Eqs. (6) and (7). Lower bounds on the $d_{16}$ elements were found to be $d_{16}=0.08 \pm 0.03 \mathrm{pmV}^{-1}$ and $0.03 \pm 0.01 \mathrm{pmV}^{-1}$, with parallel polarizers and crossed polarizers, respectively. The deviation in the $d_{16}$-coefficient between the parallel and crossed polarizers case is difficult to explain but could possibly originate from a difference in $\Delta n$ for these two cases. Nonetheless, similar values to our findings of $d_{22}$ have been reported previously. ${ }^{9,20,29}$ Equations (6) and (7) were derived under the assumption of Kleinman symmetry conditions (see appendix). The validity of Kleinman symmetry is discussed in the literature. Stoller et al. ${ }^{34}$ argue that Kleinman symmetry is valid since the SHG wavelength (400 $\mathrm{nm}$ in their case) is far from the wavelength of the first electronic transition in collagen at $310 \mathrm{~nm}$. However, Chu et al. ${ }^{18}$ stated that a $1230 \mathrm{~nm}$ laser and the resulting $615 \mathrm{~nm}$ SHG are, in fact, not far from the molecular resonant frequency of muscle fibers $(\sim 430$ and $550 \mathrm{~nm}$ ), causing a slight deviation from the Kleinman symmetry in their results. Plotnikov et al. ${ }^{35}$ also argued similarly for collagen from a rat-tail tendon. It can therefore be assumed that under the conditions described here, slight deviations from Kleinman symmetry may occur, since the generated $\mathrm{SH}$ light at $390 \mathrm{~nm}$ is quite close to the resonant molecular frequency of collagen at $350-380 \mathrm{~nm}^{36}$ The invalidity of Kleinman symmetry introduces $d_{21}$ into the $d_{\text {eff }}$ tensor in Eq. (5) and yields slightly different expressions in Eqs. (6) and (7). By fitting the approximate expressions to experimental data, the ratio $d_{16} / d_{21} \approx 0.8$ was found for both parallel and crossed polarizers. A quotient below 1 is reasonable, since an SHG beam with the electric field parallel to the fiber axis can be assumed to be more strongly resonance enhanced than an SHG beam with the electric field perpendicular to the fiber axis. Kleinman symmetry states that $d_{16}=d_{21}$ when fundamental and SHG frequencies are far from the molecular resonant frequency. ${ }^{37}$

\subsection{Comparing d-Coefficients for Malignant Melanoma and Normal Skin}

The experimental method was used on malignant melanoma and normal skin tissue to see if any differences in the $d_{22}$ parameter could be found. ROIs containing well-ordered collagen fibers were chosen because less ordered fibers in both melanoma and skin gave a poor fit of Eqs. (6) and (7) to the polar plots (data not shown). This was also the case for RTT (Fig. 6). Images of collagen and polar plots generated for the ROIs indicated in the images are shown in Fig. 9. From Eq. (8), the fit between the experimental data and Eq. (6) was 0.01 for both plots. Note that the plots are normalized and that the maximum SHG signal from skin was actually a factor four larger compared with melanoma. A significantly $(P<0.05$, $n=20,10$ ROI in 2 different sections) lower $d_{22}$-value was found in melanomas compared to normal skin, $0.053 \pm 0.003 \mathrm{pmV}^{-1}$ and $0.073 \pm 0.008 \mathrm{pmV}^{-1}$, respectively. The quotient between $d_{22}$ and $d_{16}$, which indicates the fiber's axial polarizing effects, ${ }^{9}$ was found to be approximately 1.5 and 1.8 for melanomas and normal skin, respectively. This is comparable to the value found for RTT $(\sim 1.9)$ and similar to stated values in the literature. ${ }^{22,29}$ The calculations were performed as described for RTT with necessary changes of input data due to the use of a different objective and excitation wavelength. In the case of normal skin tissue (Fig. 9), a better fit was obtained using an angle $\delta$ of $\sim 20 \mathrm{deg}$. This demonstrates the need for the more complex model, which takes into account that biopsies may be mounted in such a way that the collagen fibers are tilted an angle $\delta$ above the $x y$-plane of the glass slide.

For RTT the thickness of the sample and that of the frozen section were assumed to be the same. This may not be the case for tumors or normal tissue, where the collagen fibers are more heterogeneously distributed than in RTT. To study the impact of the sample thickness on $d_{22}$, an equivalent of Fig. 8, with input data relevant for melanoma and skin, was plotted. Minor variations in $d_{22}$ were found in the range $t_{s} \approx 2-\approx 8 \mathrm{~m}$. Thus, $t_{s}=5 \mu \mathrm{m}$ was used also in the case of skin and melanoma.

The SHG signal and $d_{22}$-coefficient depend on the structure and packing of the triple-helical collagen molecule as well as the collagen content. These factors may explain the two to three times higher $d_{22}$-coefficient in the collagen-rich and well-structured collagen fibers in RTT compared to melanoma and skin. However, more interesting is the significantly lower $d_{22}$-coefficient in malignant melanoma compared to normal skin. Collagen is the major extracellular matrix protein in human skin dermis, and the fibrils are well ordered. ${ }^{8,38}$ Previous biochemical measurements of collagen content in skin and human osteosarcoma revealed 10-15 times more collagen in skin, ${ }^{39}$ and the osteosarcoma xenograft has been found to contain more collagen than the melanoma FME used in the present study. ${ }^{40}$ In accordance with this, the SHG images of the collagen fibers (Fig. 9) clearly demonstrate the abundance of collagen fibers in the skin dermis compared to malignant melanoma. The intensity of the SHG signal was also higher in skin compared to melanoma. Basal cell carcinoma has also been found to exhibit a decreased SHG signal compared to normal dermal stroma. ${ }^{16}$ The present work demonstrates the potential of using the SHG signal quantified by 
the $d_{22}$-coefficient as an optical biomarker to discriminate between normal and malignant tissue.

\section{Summary and Conclusions}

SHG imaging is gradually finding its place as a research and diagnostic tool in biology and medicine. The work described in this paper contributes to this development by presenting a model and a method designed for obtaining both data and images of SHG signals from tissues containing collagen. The method is based upon previously described experiments in the literature but is extended to also include analysis of fibers tilted an angle $\delta$ above the $x y$-plane. The method also differs in that a laser scanning microscope is employed for image acquisition and data analysis. The images obtained are fundamental to the data analysis, and the potent tool this imaging modality provides is especially significant when considering which areas in the image to analyze. For instance, by selecting different regions of interest, it was possible to compare ordered and less ordered collagen fibers in any specific image. It was found that ordered collagen fibers could be well described by the presented models. Lower bounds for the second-order susceptibility in collagen were determined to be $d_{22} \geqslant 0.15 \mathrm{pmV}^{-1}$ and $d_{16} \geqslant 0.08 \mathrm{pmV}^{-1}$. Slight deviations from Kleinman symmetry conditions were observed and quantified. Significant differences in the values of $d_{22}$ were found between a human melanoma tumor and comparable normal mouse skin tissue. The findings were $0.053 \pm 0.003 \mathrm{pmV}^{-1}$ and $0.073 \pm 0.008 \mathrm{pmV}^{-1}$ for tumor and skin, respectively. The results are encouraging and open up for SHG analysis of structure, orientation, and nonlinear behavior in normal and pathological tissues.

\section{Acknowledgments}

This work was supported by the Norwegian Research Council and the Norwegian Cancer Society. The authors are grateful for the help from Ingunn Tufto (Department of Physics, the Norwegian University of Science and Technology) in obtaining the tissue samples, and the frozen sections were kindly prepared at the Department of Pathology, St. Olav's Hospital.

\section{Appendix: Derivation of Eqs. (6) and (7)}

An expression for the d-tensor, $d_{i j k}^{\prime}$, in a transformed coordinate system rotated an angle $\delta$ about the $x$-axis can be found as follows. The rotation matrix is

$$
R_{x}=\left(\begin{array}{ccc}
1 & 0 & 0 \\
0 & \cos \delta & \sin \delta \\
0 & -\sin \delta & \cos \delta
\end{array}\right)=\left(\begin{array}{lll}
l_{11} & l_{12} & l_{13} \\
l_{21} & l_{22} & l_{23} \\
l_{31} & l_{32} & l_{33}
\end{array}\right),
$$

and the appropriate transformation of the second-rank d-tensor is given by

$$
d_{i j k}^{\prime}=l_{i p} l_{j q} l_{k r} d_{p q r}
$$

where $d_{p q r}$ in contracted notation becomes [Eq. (5) using cylindrical and Kleinman symmetry]

$$
\left[\begin{array}{cccccc}
0 & 0 & 0 & 0 & 0 & d_{16} \\
d_{16} & d_{22} & d_{16} & 0 & 0 & 0 \\
0 & 0 & 0 & d_{16} & 0 & 0
\end{array}\right]
$$

Using Eqs. (10)-(12), we can find the matrix elements of $d_{i j k}^{\prime}$ as

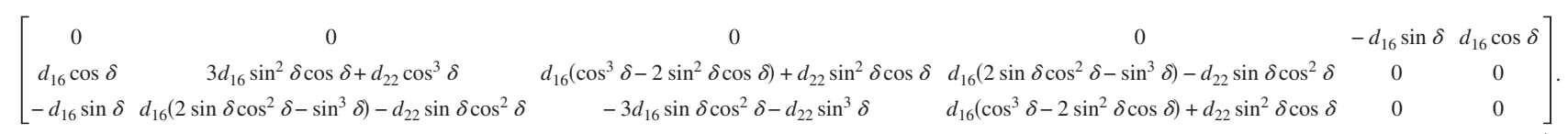

For the case of parallel polarizers, the unit vectors describing pump and associated SHG fields are

$$
\hat{e}_{\omega}=\hat{e}_{2 \omega}=(-\sin \beta, \cos \beta, 0)
$$

For the case of crossed polarizers, the unit vectors describing the pump and SHG fields are

$$
\begin{gathered}
\hat{e}_{\omega}=(-\sin \beta, \cos \beta, 0) \\
\hat{e}_{2 \omega}=(-\cos \beta,-\sin \beta, 0)
\end{gathered}
$$

Substitution of Eq. (13) in Eq. (5) and using the appropriate expressions for the unit vectors, Eqs. (6) and (7) are found by matrix multiplication.

\section{References}

1. W. R. Zipfel, R. M. Williams, and W. W. Webb, "Nonlinear magic: multiphoton microscopy in the biosciences," Nat. Biotechnol. 21(11), 1368-1376 (2003).

2. W. G. Wang, J. B. Wyckoff, V. C. Frohlich, Y. Oleynikov, S. Huttelmaier, J. Zavadil, L. Cermak, E. P. Bottinger, R. H. Singer, J. G White, J. E. Segall, and J. S. Condeelis, "Single cell behavior in metastatic primary mammary tumors correlated with gene expression patterns revealed by molecular profiling," Cancer Res. 62(21), 62786288 (2002).

3. L. Eikenes, O. S. Bruland, C. Brekken, and C. de L. Davies, "Collagenase increases the transcapillary pressure gradient and improves the uptake and distribution of monoclonal antibodies in human osteosarcoma xenografts," Cancer Res. 64(14), 4768-4773 (2004).

4. P. A. Netti, D. A. Berk, M. A. Swartz, A. J. Grodzinsky, and R. K. Jain, "Role of extracellular matrix assembly in interstitial transport in solid tumors," Cancer Res. 60(9), 2497-2503 (2000).

5. P. J. Campagnola, A. C. Millard, M. Terasaki, P. E. Hoppe, C. J. Malone, and W. A. Mohler, "Three-dimensional high-resolution second harmonic generation imaging of endogenous structural proteins in biological tissues," Biophys. J. 81(1), 493-508 (2002). 
6. P. Stoller, K. M. Reiser, P. M. Celliers, and A. M. Rubenchik, "Polarization-modulated second harmonic generation in collagen," Biophys. J. 82(6), 3330-3342 (2002).

7. E. Brown, T. McKee, E. diTomaso, A. Pluen, B. Seed, Y. Boucher, and R. K. Jain, "Dynamic imaging of collagen and its modulation in tumors in vivo using second-harmonic generation," Nat. Med. 9(6), 796-800 (2003).

8. T. Yasui, Y. Tohno, and T. Araki, "Characterization of collagen orientation in human dermis by two-dimensional second-harmonicgeneration polarimetry," J. Biomed. Opt. 9(2), 259-264 (2004).

9. R. M. Williams, W. R. Zipfel, and W. W. Webb, "Interpreting secondharmonic generation images of collagen I fibrils," Biophys. J. 88(2), 1377-1386 (2005)

10. R. W. Boyd, Nonlinear Optics, Academic Press, San Diego, CA (1992).

11. A. Zoumi, A. Yeh, and B. J. Tromberg, "Imaging cells and extracellular matrix in vivo by using second-harmonic generation and twophoton excited fluorescence," Proc. Natl. Acad. Sci. U.S.A. 99(17), 11014-11019 (2002).

12. P. J. Campagnola, A. C. Millard, M. Terasaki, P. E. Hoppe, C. J. Malone, and W. A. Mohler, "Three-dimensional high-resolution second-harmonic generation imaging of endogenous structural proteins in biological tissues," Biophys. J. 82(1), 493-508 (2002).

13. L. Moreaux, O. Sandre, S. Charpak, M. Blanchard-Desce, and J. Mertz, "Coherent scattering in multi-harmonic light microscopy," Biophys. J. 80(3), 1568-1574 (2001).

14. G. Cox, E. Kable, A. Jones, I. K. Fraser, F. Manconi, and M. D. Gorrell, "3-dimensional imaging of collagen using second harmonic generation,” J. Struct. Biol. 141(1), 53-62 (2003).

15. N. D. Kirkpatrick, J. B. Hoying, S. K. Botting, J. A. Weiss, and U. Utzinger, "In vitro model for endogenous optical signatures of collagen," J. Biomed. Opt. 11(5), 054021-1-8 (2006).

16. S. J. Lin, S. H. Jee, C. J. Kuo, R. J. Wu, W. C. Lin, J. S. Chen, Y. H. Liao, C. J. Hsu, T. F. Tsai, Y. F. Chen, and C. Y. Dong, "Discrimination of basal cell carcinoma from normal dermal stroma by quantitative multiphoton imaging," Opt. Lett. 31(18), 2756-2758 (2006)

17. W. R. Zipfel, R. M. Williams, R. Christie, A. Y. Nikitin, B. T. Hyman, and W. W. Webb, "Live tissue intrinsic emission microscopy using multiphoton-excited native fluorescence and second harmonic generation," Proc. Natl. Acad. Sci. U.S.A. 100(12), 7075-7080 (2003).

18. S. W. Chu, S. Y. Chen, G. W. Chern, T. H. Tsai, Y. C. Chen, B. L. Lin, and C. K. Sun, "Studies of $\mathrm{x}((2)) \mathrm{x}((3))$ tensors in submicronscaled bio-tissues by polarization harmonics optical microscopy," Biophys. J. 86(6), 3914-3922 (2004).

19. K. M. Tveit, O. Fodstad, J. V. Johannessen, and S. Olsnes, "A humanmelanoma cell-line established from xenograft in athymic mice," $\mathrm{Br}$. J. Cancer 41(5), 724-733 (1980).

20. P. Stoller, P. M. Celliers, K. M. Reiser, and A. M. Rubenchik, "Quantitative second-harmonic generation microscopy in collagen," Appl. Opt. 42(25), 5209-5219 (2003).

21. J. Örtegren, Liquid Crystalline Polymers for Nonlinear Optics: Pyroelectrical Polymers and Ferroelectric Dendrimers, Royal Institute of Technology, Stockholm (2001).

22. P. Stoller, B. M. Kim, A. M. Rubenchik, K. M. Reiser, and L. B. Da Silva, "Polarization-dependent optical second-harmonic imaging of a rat-tail tendon," J. Biomed. Opt. 7(2), 205-214 (2002)
23. M. Born and E. Wolf, Principles of Optics, Pergamon Press, Oxford (1993).

24. B. Eyden and M. Tzaphlidou, "Structural variations of collagen in normal and pathological tissues: role of electron microscopy," $\mathrm{Mi}$ cron 32(3), 287-300 (2001).

25. M. W. K. Chew and J. M. Squire, "Cryosections of x-ray monitored collagen fibrils provide support for quasi-hexagonal molecular packing," Int. J. Biol. Macromol. 8(1), 27-36 (1986).

26. D. A. D. Parry and A. S. Craig, "Quantitative electron-microscope observations of collagen fibrils in rat-tail tendon," Biopolymers 16(5), 1015-1031 (1977).

27. D. J. S. Hulmes, "Building collagen molecules, fibrils, and suprafibrillar structures," J. Struct. Biol. 137(1-2), 2-10 (2002).

28. S. Roth and I. Freund, "Optical 2nd-harmonic scattering in rat-tail tendon,” Biopolymers 20(6), 1271-1290 (1981).

29. I. Freund, M. Deutsch, and A. Sprecher, "Connective-tissue polarity-optical 2nd-harmonic microscopy, crossed-beam summation, and small-angle scattering in rat-tail tendon," Biophys. J. 50(4), 693-712 (1986).

30. V. G. Dmitriev, G. G. Gurzadyan, and D. N. Nikogoyan, Handbook of Nonlinear Optical Crystals, Springer, Berlin (1997).

31. F. P. Bolin, L. E. Preuss, R. C. Taylor, and R. J. Ference, "Refractiveindex of some mammalian-tissues using a fiber optic cladding method," Appl. Opt. 28(12), 2297-2303 (1989).

32. D. T. Poh, "Examination of refractive index of human epidermis invitro," in Proc., Int. Conf. Lasers "96, V. J. Corcoran and T. A. Goldman, eds., pp. 118-125, STS, McLean, VA (1997).

33. T. Gutsmann, G. E. Fantner, M. Venturoni, A. Ekani-Nkodo, J. B. Thompson, J. H. Kindt, D. E. Morse, D. K. Fygenson, and P. K. Hansma, "Evidence that collagen fibrils in tendons are inhomogeneously structured in a tubelike manner," Biophys. J. 84(4), 25932598 (2003)

34. P. Stoller, K. M. Reiser, P. M. Celliers, and A. M. Rubenchik, "Polarization-modulated second harmonic generation in collagen," Biophys. J. 82(6), 3330-3342 (2002).

35. S. V. Plotnikov, A. C. Millard, P. J. Campagnola, and W. A. Mohler, "Characterization of the myosin-based source for second-harmonic generation from muscle sarcomeres," Biophys. J. 90(2), 693-703 (2006).

36. A. Katz and R. R. Alfano, "Noninvasive fluorescence-based instrumentation for cancer and precancer detection and screening," in Proc. of SPIE, G. E. Cohn, ed., 3931, pp. 223-226 (2000).

37. D. A. Kleinman, "Nonlinear dielectric polarization in optical media," Phys. Rev. 126(6), 1977-1979 (1962)

38. T. Yasui, Y. Tohno, and T. Araki, "Determination of collagen fiber orientation in human tissue by use of polarization measurement of molecular second-harmonic-generation light," Appl. Opt. 43(14), 2861-2867 (2004)

39. C. de L. Davies, B. O. Engesaeter, I. Haug, I. W. Ormberg, J. Halgunset, and C. Brekken, "Uptake of IgG in osteosarcoma correlates inversely with interstitial fluid pressure, but not with interstitial constituents," Br. J. Cancer 85(12), 1968-1977 (2001).

40. C. de L. Davies, H. Muller, I. Hagen, M. Garseth, and M. H. Hjelstuen, "Comparison of extracellular matrix in human osteosarcomas and melanomas growing as xenografts, multicellular spheroids, and monolayer cultures," Anticancer Res. 17(6D), 4317-4326 (1997). 